УДК 669.717:629.4.027.4

\title{
Effect of Precipitation with Core-Shell Structure on Mechanical Properties of PN-EN 2024 T6I6 Alloy
}

\author{
Hanna Radziszewska $a^{a^{*}}$, \\ Lukas Kaczmarek $^{\mathrm{b}}$ and Zbigniew Gawroński ${ }^{\mathrm{b}}$ \\ ${ }^{a}$ Youth Educational Centre \\ 151 Drewnowska Str., Łódź, 91-008, Poland \\ ${ }^{b}$ Technical University of Lodz \\ 1/15 Stefanowskiego Str., Łódź, 90-924, Poland
}

Received 28.02.2017, received in revised form 10.03.2017, accepted 23.03.2017

The article provides results of investigation of aluminium alloy PN-EN 2024 in condition T6I6 and compossibility the kinetic mechanism of precipitation formation with existing theories of precipitation with core-shell structure formation. Also the results of estimation of these precipitation influence on increasing of strength, fatigue and ductility are presented.

Keywords: aluminium alloys, ductility, strengthening, fatigue.

DOI: $10.17516 / 1998-2836-0007$.

(C) Siberian Federal University. All rights reserved

* Corresponding author E-mail address: hanka54@poczta.fm 


\title{
Влияние выделений \\ со структурой сердечник/оболочка \\ на механические свойства сплава PN-EN 2024 T6I6
}

\author{
Ханна Радзишевска ${ }^{a}$, \\ Лукаш Качмарек ${ }^{0}$, Збигнев Гавронски \\ ${ }^{a} Ц$ центр Воспитания Молодёжи \\ Польша, 91-008., Лодзь, Drewnowska Str., 151 \\ ${ }^{б}$ Технический университет г. Лодзь \\ Польша, 90-924, Лодзь, Stefanowskiego Str., 1/15
}

В работе показаны результаты исследования алюминиевого сплава PN-EN 2024 в состоянии T6I6 и сопоставимости кинетического механизма образования выделений с существующими гипотезами формирования выделений со структурой сердечник/оболочка. Также показаны результаты оценки влияния этих выделений на повышение прочности, усталости и одновременно пластичности материала.

Ключевые слова: алюминиевые сплавы, пластичность, упрочнение, усталость.

\section{Введение}

Металлографические лаборатории по всему миру находятся в поисках новых технологических решений по достижению максимально возможной производительности коммерческих промышленных сплавов. Полученные результаты подтверждают, что для повышения свойств алюминиевых сплавов необходимо изменять химический состав и использовать современные методы пластической деформации SPD (Severe Plastic Deformation) и термической обработки для получения ультрамелкокристаллической структуры. Используемые в последнее время стандартные способы термообработки Т3, Т6, Т7 позволяют повысить только одну характеристику промышленного сплава, а остальные, как правило, снижаются. Например, сплавы серии 7xxx, модифицированные скандием и цирконием (АА7085), имеют мелкозернистую структуру, а следовательно, высокую прочность, но низкую трещиностойкость [1].

В алюминиевых сплавах с литием второго и третьего поколения за счёт одновременного использования современных методов термообработки и пластической деформации были получены повышенные в значительной степени свойства [2-4]. В этом случае значительное упрочнение материала обусловливается мелкозернистой структурой, полученной при пластической деформации, и выделений, образующихся во время термообработки, в составе которых присутствуют серебро, литий, цирконий и скандий. Следует отметить, что сплавы с литием третьего поколения являются относительно дорогими и их производство требует специальных условий. Из литературных данных известно, что у многих промышленных сплавов повышение механических свойств связано с использованием современных методов SPD, которые приводят к получению наноструктуры. Тем не менее до сих пор эти методы являются полупромышлен- 
ными, высокоэнергетическими, неавтоматическими и, прежде всего, могут быть применены только для материалов с определенной пластичностью $[5,6]$.

Значительное повышение прочности вследствие изменения химического состава достигается при использовании в качестве легирующих элементов скандия, циркония и лития. Отмечена возможность образования выделений $\mathrm{Al}_{3} \mathrm{Sc}, \mathrm{Al}_{3} \mathrm{Zr}, \mathrm{Al}_{3} \mathrm{Li}$ и композитных фаз $\mathrm{Al}_{3}\left(\mathrm{~A}_{1-\mathrm{x}}\right.$ ${ }_{\mathrm{y}} \mathrm{B}_{\mathrm{x}} \mathrm{C}_{\mathrm{y}}$ ), где $\mathrm{A}, \mathrm{B}, \mathrm{C}$ - другие легирующие элементы, имеющие кристаллическую решетку по типу $\mathrm{Ll}_{2}$ [7-13]. Было также отмечено, что наибольшее упрочнение сплава наблюдается, когда эти фазы кристаллизуются гетерогенно с образованием выделений со структурой сердечник/ оболочка $[7,14]$. На основе лабораторных исследований определены условия, необходимые для получения выделений со структурой сердечник/оболочка $[10,11]$. Также были предложены две гипотезы кинетики механизма образования такого выделения. Первая предполагает формирование оболочки на ранее сформированном сердечнике - фазы типа $\mathrm{Al}_{3} \mathrm{~B}\left(\mathrm{L1}_{2}\right)[11,15-17]$. Вторая гипотеза предполагает, что образование гетерогенного выделения типа $\mathrm{Al}_{3}\left(\mathrm{~A}_{1-\mathrm{x}} \mathrm{B}_{\mathrm{x}}\right)$ происходит с образованием сердечника и оболочки путем диффузии внутри выделения и за счёт замены атомов в узлах кристаллической решётки $[8,14,18]$.

В результате испытаний было отмечено, что гетерогенная кристаллизация возможна только при помощи многостадийной термообработки. Применение сегментной термообработки RRA (Retrogression and Re-ageing) и НTPP (High-temperature pre-precipitation) в сочетании со стандартной пластической деформацией $[1,19,20]$ зачастую позволяет одновременно упрочнить сплав за счет образования зон ГП (Guinier-Preston`a), ГПБ (Guinier-Preston-Bagaryatsky`a) и метастабильных фаз, образующихся в зёрнах во время первичного старения, а также повысить устойчивость к коррозии благодаря толстым выделениям равновесной фазы на границах зёрен во время окончательного старения [20]. Изменение режимов обработки обеспечивает разнообразие кинетики нуклеации выделений и получение значительных различий между выделениями внутри зёрен и на их границах. Использование обработки с перерывом первичного старения путем охлаждения до комнатной температуры (режим «І») подтверждает возможность получения более высокой максимальной твёрдости и максимального упрочнения за меньшее время и без снижения твёрдости по сравнению со стандартной обработкой. Кроме того, эти режимы могут быть объединены с различными режимами пластической деформации [21, 22]. Наиболее эффективной является обработка по режиму Т6I6, с помощью которой можно повысить твёрдость, предел текучести и сопротивление растяжению по сравнению со стандартными режимами обработки Т3, Т6 и даже Т8 или Т9.

В литературе описаны испытания, проведенные только для лабораторных сплавов, и не представлены результаты о возможности получения выделений со структурой сердечник/оболочка в промышленных сплавах. В Институте материаловедения Технического университета г. Лодзи после обработки алюминиевого сплава PN-EN 2024 по режиму Т6I6 были получены уникальные механические свойства. Во время предварительных исследований в микроструктуре сплава PN-EN 2024 в состоянии T6I6 обнаружен новый тип двухфазных выделений со структурой, состоящей из сердечника и оболочки. Химический состав сплава, главным образом легирующие элементы (медь и марганец), способствуют созданию условий для образования двухфазного выделения. Поэтому целью данного исследования является изучение сопоставимости кинетического механизма образования выделений с существующими гипотезами 
формирования выделений сердечник/оболочка, а также исследование влияния этих выделений на упрочнение и пластичность материала.

\section{Методика проведения исследования}

Исследовали сплав PN-EN 2024 системы Al-Cu-Mg. Химический состав сплава определен с помощью рентгеновского спектроскопа Siemens SRS 303, сплав содержит (\% мас.): 4,5 Cu; 1,37 $\mathrm{Mg} ;$ 0,61 Mn; 0,07 Si; 0,27 Fe; 0,02 Zn; 0,02 Ti, остальное - алюминий. Все исследуемые образцы взяты из одного прутка диаметром 30 мм в состоянии Т3 и подвергнуты термической обработке по режиму Т6I6 (табл. 1).

Морфология и фазовый состав исследованы на сканирующем электронном микроскопе (CЭM) Hitachi S-3000N, степень дисперсности фаз определена по программе Met-Ilo v.5.1. Микроструктура сплава и выделений со структурой сердечник/оболочка исследованы на световом микроскопе Neophot-32. Химический состав выделений установлен с помощью сканирующего трансмиссионного электронного микроскопа (СТЭМ) Hitachi S5500 с рентгеновским микроанализатором ЭДС (Energy Dispersive Spectroscopy). Образцы для исследований были получены путем механического среза с помощью Dimple Grynder Model Gatan Model 656. Тонкие пленки получены путем ионного утонения на приборе Precision Ion Polishing System 691 Gatan Inc. Kроме того, использование сканирующего ионного микроскопа с FIB (Focused ion Beam) позволило подготовить и предварительно исследовать пленки перед проведением анализа с применением ТЭМ, СЭМ и рентгеновского микроанализа.

Испытание на усталость проведено с использованием электродинамического возбудителя Guide to Applied Mechanics' Vibration Test System Operation согласно инструкции. После определения собственной частоты свободного конца образца (ping test) проведена калибровка каждого анализируемого образца в диапазоне до 110 МПа (три косвенных измерения). Затем соответствующие усталостные испытания были проведены при требуемом уровне напряжений с контролем отклонения свободного конца образца. Тесты проводили на шести уровнях напря-

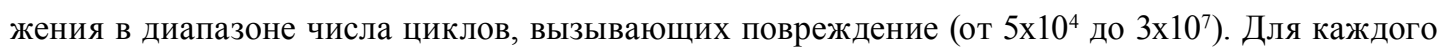
уровня напряжений были исследованы четыре образца, в общей сложности 24. Момент, когда произошло резкое падение, стабильной до этого частоты примерно на $1 \%$, был принят как начало резкого падения частоты. Морфология и структура фазового состава исследованы на сканирующем электронном микроскопе (СЭМ) Hitachi S-3000. Полученные результаты были использованы для выявления предела усталостной прочности $Z_{\mathrm{go}}$. Анализ микроструктуры изломов и морфологии упрочняющих выделений сердечник/оболочка позволили определить влияние последних на зарождение и распространение усталостных трещин в алюминиевом сплаве PN-EN 2024 в состоянии Т6I6 при приложении переменных нагрузок.

Таблица 1. Параметры термообработки сплава PN-EN 2024 по режиму T6I6

\begin{tabular}{|c|c|c|c|}
\hline \multirow{2}{*}{ Режим обработки } & \multirow{2}{*}{$\begin{array}{c}\text { Температура } \\
\text { и время обработки }\end{array}$} & \multicolumn{2}{|c|}{ Температура и время старения } \\
\cline { 3 - 4 } & I этап & II этап \\
\hline T6I6 & $773 \mathrm{~K} ; 16 \mathrm{~h}$ & $393 \mathrm{~K} ; 1,5 \mathrm{~h}$ & $433 \mathrm{~K} ; 6 \mathrm{~h}$ \\
\hline
\end{tabular}

$$
-70-
$$


Испытания на растяжение проведены на машине Instron 35 с сенсометром, работающим в диапазоне нагрузки $0,5 \kappa \mathrm{H} ; 1,25 \kappa \mathrm{H}, 5 \kappa \mathrm{H}$ и со скоростью от 0,001 до 3000 мм/мин. Испытания осуществлены в соответствии с польским стандартом, для этого были вырезаны по восемь образцов в радиальном и в осевом направлении. Основываясь на полученных данных, был установлен предел прочности при растяжении.

\section{Результаты исследования выделений}

Анализ микроструктуры сплава PN-EN 2024 в состоянии T6I6 показал наличие выделений размером 1-3 мкм со структурой сердечник/оболочка, формирование которых зависит от параметров второй стадии старения (рис. 1). Согласно теории кристаллизации в процессе первичного старения образуются фазы с различным химическим составом и размером. В первую очередь кристаллизуют фазы, состоящие из элементов с относительно высоким коэффициентом диффузии в $\mathrm{Al}$ и относительно высокой энергетической связью между ними $(\mathrm{Mg}$, $\mathrm{Cu}$ ), что приводит к образованию стабильных упрочняющих фаз (S-фаза, $\theta$-фаза). Тем не менее внезапное прерывание процесса первичного старения (режим T6I6) ограничивает диффузию и упорядочивает расположение атомов в узлах кристаллической решетки образующихся упрочняющих фаз. Кроме того, внутри образующихся зон ГП и ГПБ находятся атомы других легирующих элементов (Mn, Fe), которые имеют относительно низкий коэффициент диффузии в Al. Во время вторичного старения при более высокой температуре, чем при первичном, происходит гетерогенная кристаллизация, при которой на первичных стабильных выделениях образуется оболочка из атомов элементов с относительно низким коэффициентом диффузии в Al; она является слоем ингибирования, так как замедляет диффузию элементов сердечника этого выделения. Этот процесс образования выделения в сплаве PN-EN 2024 в состоянии Т6I6 соответствует второму механизму образования гетерогенного выделения со структурой сердечник/оболочка (рис. 1).

На рис. 2 показано изображение анализируемого выделения (СТЭМ) и выделены области, которые в дальнейшем были исследованы с помощью рентгеновской микроскопии и в них определен химический состав светлой и темной фаз (Base 8); в них также определены морфологии и карты расположения атомов элементов СЭ химического состава светлой и темной фаз (Base 7, Base 11); с помощью сканирующего ионного микроскопа исследован химический состав светлой и темной фаз (Base 9, Base 10, Base 12, Base 13).

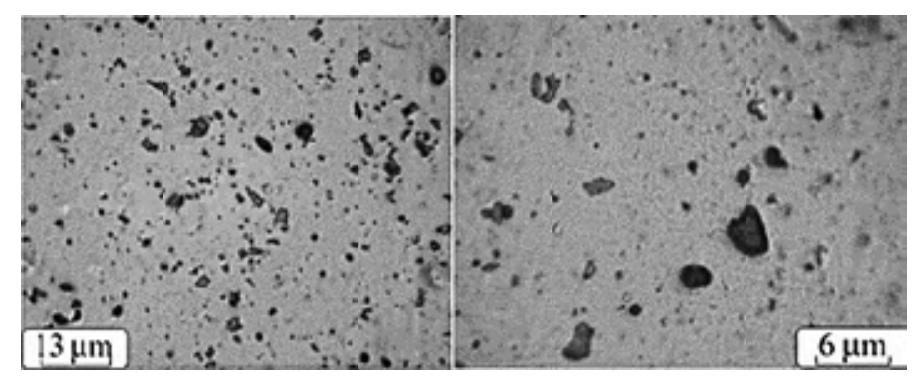

Рис. 1. Микроструктура сплава PN-EN 2024 в состоянии T6I6 с частицами выделений со структурой сердечник/оболочка при разном увеличении 
На рис. 3-5 представлены результаты спектра ЭДС, химический состав и профиль концентрации атомов легирующих элементов $\mathrm{Cu}, \mathrm{Fe}, \mathrm{Si}, \mathrm{Mn}$ в областях Base 8 и Base 9.

На следующем этапе исследования проведен анализ морфологии и карт СЭ химического состава светлой и темной фаз в областях Base 7 и Base 11. В этом случае особое внимание было уделено распределению элементов, расположенных вдоль границы светлая - темная фаза. Результаты наблюдения области Base 7 показаны на рис. 6.

После анализа карт распределения элементов в основе и в выделениях можно сделать вывод о том, что они имеют химический состав, отличный от фазы $\mathrm{S}$ и фазы $\theta$, обычно возникающих в сплавах системы $\mathrm{Al}-\mathrm{Cu}-\mathrm{Mg}$ последовательно при упрочнении. Проведен качественный анализ химического состава фаз, оценена доля каждого элемента, по усред-

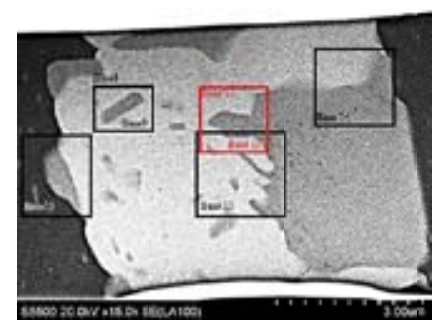

Рис. 2. СТЭМ, сплав PN-EN 2024 в состоянии Т6I6, с областями Base 7, Base 8, Base 9, Base 10, Base 11, Base 12, Base 13
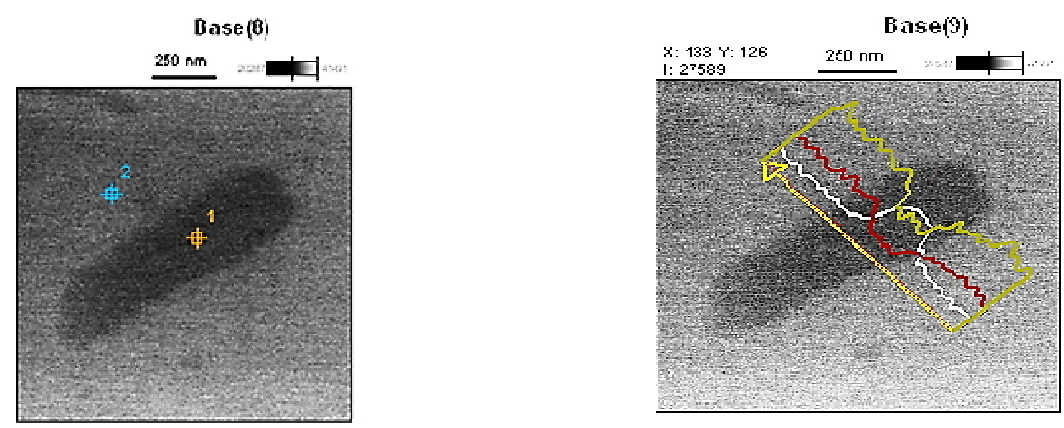

Рис. 3. СТЭМ, сплав PN-EN 2024 в состоянии T6I6, область Base 8 и Base 9 внутри выделения
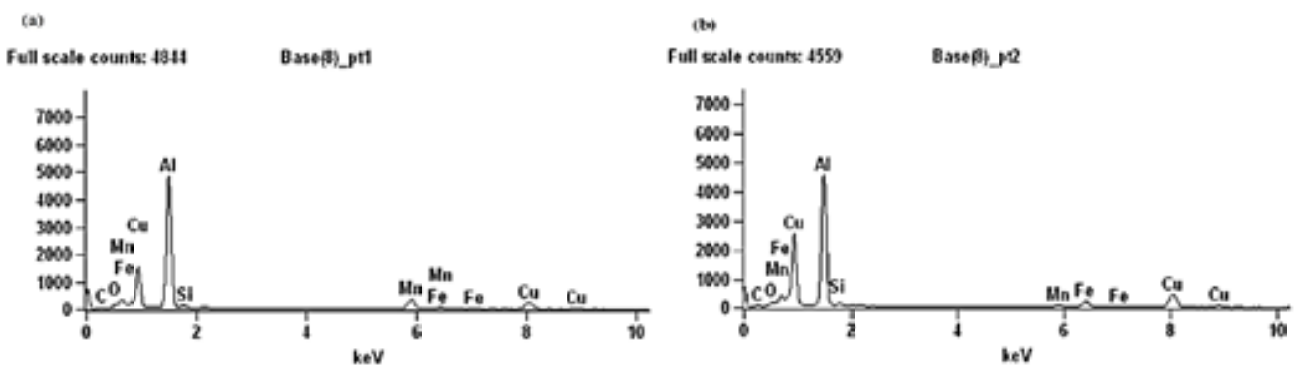

Рис. 4. Спектр ЭДС, рентгеновский микроанализ, выделение со структурой сердечник/оболочка в области Base 8: pt1 - темная фаза; pt2 - светлая фаза 

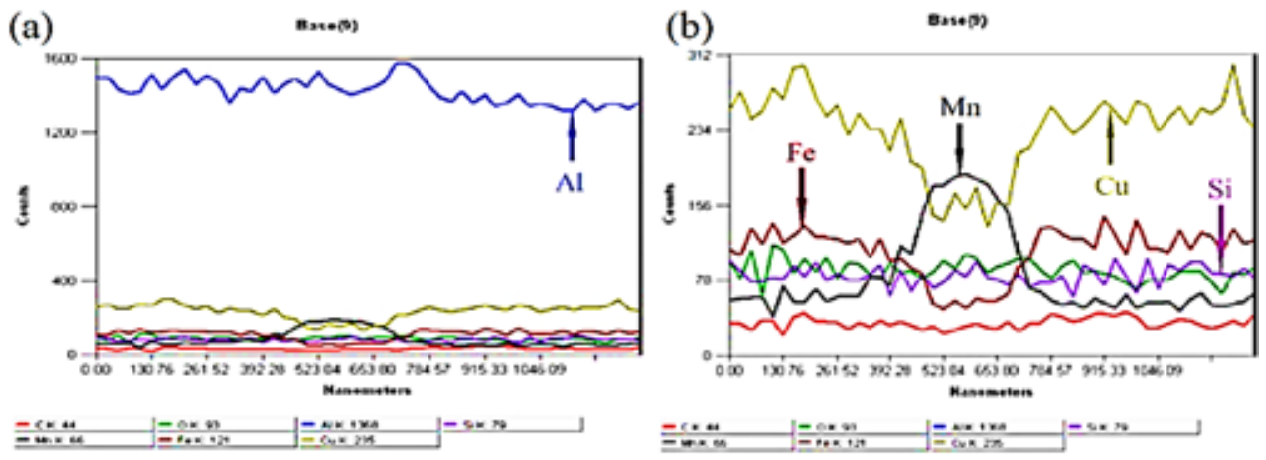

Рис. 5. Профиль концентрации атомов легирующих элементов $\mathrm{Cu}, \mathrm{Fe}, \mathrm{Si}, \mathrm{Mn}$ в области Ваse 9 при разном увеличении. Красная линия на обеих диаграммах обозначает концерентацию атомов углерода

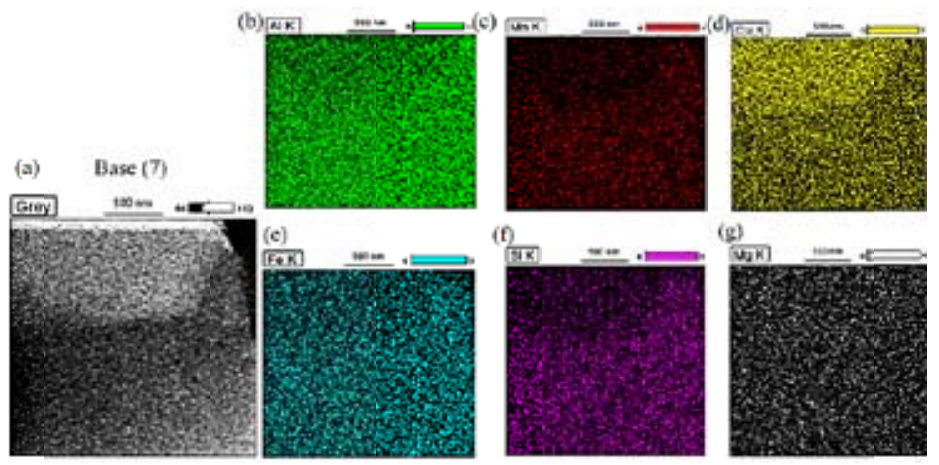

Рис. 6. Карты СЭ Base 7; (а) граница основа/выделение; (b) Al; (c) Mn; (d) Cu; (e) Fe; (f) $\mathrm{Si}$ (g) $\mathrm{Mg}$

ненным результатам определено, что соединение $-\mathrm{Al}_{3} \mathrm{~B}$, где В представляет собой $\left(\mathrm{Cu}_{\mathrm{x}}, \mathrm{Fe}_{\mathrm{y}}\right.$, $\left.\mathrm{Mn}_{1-\mathrm{x}-\mathrm{y}}\right)$.

Основываясь на результатах исследований и теориях о формировании выделений со структурой сердечник/оболочка, можно выдвинуть гипотезу о кинетике формирования этих выделений в материале, связанной с сегрегацией легирующих элементов в рамках выделения (второй механизм). В соответствие с предположениями процесс образования выделения можно разбить на четыре этапа.

Этап быстрого зарождения, вероятно, начинается с роста зон ГП(Al-Cu) и ГПБ(Al-Mg$\mathrm{Cu}$ и п превращения этих зон в фазы $\theta, \theta$, $\mathrm{S}, \mathrm{S}$ '. Они являются выделениями манометрических размеров, вокруг которых кристаллизуется фаза $\mathrm{Al}_{3}\left(\mathrm{Cu}_{\mathrm{x}}, \mathrm{Fe}_{y}, \mathrm{Mn}_{1-\mathrm{x}-\mathrm{y}}\right)$. В то же время внутри выделения простые фазы $\left(\mathrm{Al}_{3} \mathrm{Cu}, \mathrm{Al}_{3} \mathrm{Fe}\right.$ и $\left.\mathrm{Al}_{3} \mathrm{Mn}\right)$ не наблюдаются, что подтверждает гипотезу о второй кинетике механизма формирования выделения со структурой сердечник/оболочка. Во время первичного старения при температуре 393 К, вероятно, происходит второй этап быстрого роста разделения. Атомы меди, железа и марганца диффундируют в центр выделения, а периферия обедняется по этим элементам (рис. $6 b$ ). На этом этапе также отмечена диффузия атомов кремния и магния из выделения в твердый раствор $\alpha$, что следует из сравнения их концентраций в выделении и матрице. Более высокая концентрация атомов меди в центральной ча- 
сти выделения объясняется более высокой скоростью диффузии в алюминии $\left(\mathrm{D}_{\mathrm{o}}=1,6 \times 10^{-6} \mathrm{~m}^{2} / \mathrm{c}\right)$, а замедление диффузии атомов марганца в выделение связано с более низкой скоростью диффузии этих атомов в алюминии $\left(\mathrm{D}_{\mathrm{o}}=8,7 \times 10^{-3} \mathrm{~m}^{2} / \mathrm{c}\right)$, что становится причиной более высокой его концентрации во внешнем слое и служит препятствием для дальнейшей диффузии атомов меди и железа. Существует также явление задержки атомов $\mathrm{Cu}$ и Fе внутри выделения. В связи с тем, что атомы $\mathrm{Cu}, \mathrm{Fe}$ и $\mathrm{Mn}$, которые могли бы при этой температуре диффундировать внутри выделения, не находятся в непосредственной близости в матрице, этап роста выделения заканчивается. Кроме того, высокое содержание Мn во внешнем слое уменьшает несоответствие решётки фазы $\mathrm{Al}_{3}\left(\mathrm{Cu}_{\mathrm{x}} \mathrm{Fe}_{\mathrm{y}} \mathrm{Mn}_{1-\mathrm{-x}-\mathrm{y}}\right)$ с решеткой фазы $\mathrm{Al}_{3} \mathrm{Cu}$ из-за разницы в валентных радиусах: $\mathrm{Mn}-139 п \mathrm{~m} ; \mathrm{Fe}-125 п \mathrm{~m} ; \mathrm{Cu}-161 п \mathrm{~m}$. Это может иметь положительное воздействие на стабильность выделений, достигающих относительно большие размеры (до 2 мкм). Во время вторичного старения при температуре $433 \mathrm{~K}$ внутри выделения создается градиент концентрации атомов $\mathrm{Cu}, \mathrm{Fe}$ и $\mathrm{Mn}$. Существует вероятность превращения атомов $\mathrm{Cu}, \mathrm{Fe}$ и $\mathrm{Mn}$ в узлах решётки, протекания диффузии атомов $\mathrm{Cu}$ и $\mathrm{Fe}$ в центр, а атомов $\mathrm{Mn}$ - к периферии выделения: происходит этап формирования оболочки.

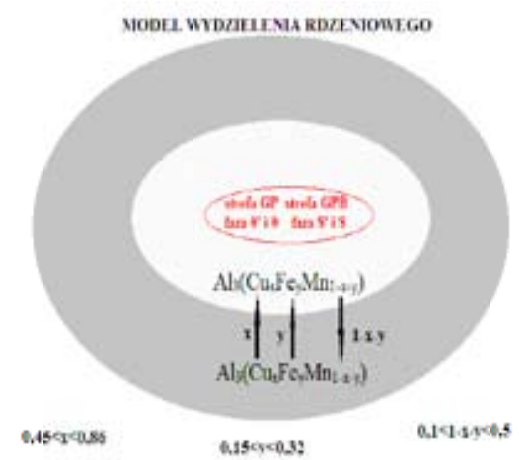

Рис. 7. Модель кинетики образования выделения со структурой сердечник/оболочка в алюминиевом сплаве PN-EN 2024 в состоянии T6I6; изменение величины коэффициентов х; у; 1-х-у указывает на изменение концентрации атомов $\mathrm{Cu}, \mathrm{Fe}$ и $\mathrm{Mn}$ в выделении

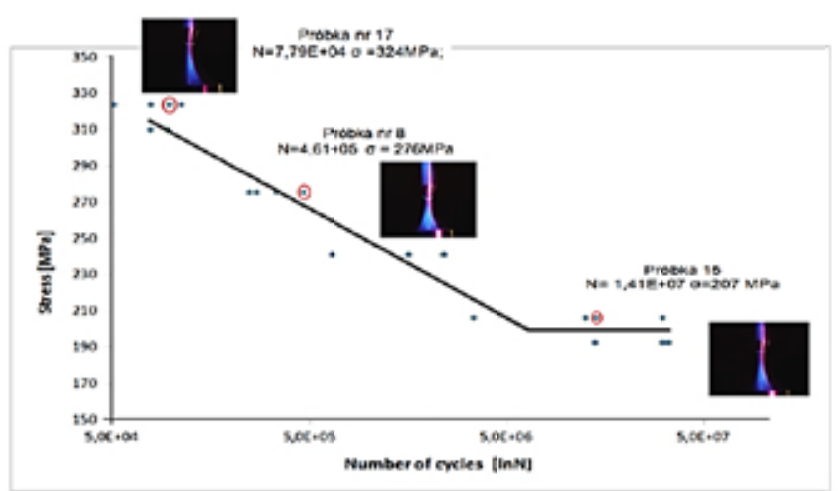

Рис. 8. Кривая Wöhler'a; зависимость максимальных напряжений от числа циклов $\sigma=\mathrm{f}(\mathrm{N})$ резонансных испытаний на усталость образцов алюминиевого сплава PN-EN 2024 в состоянии Т6I6 
На рис. 7. представлена модель кинетики образования выделения со структурой сердечник/оболочка в исследуемом алюминиевом сплаве PN-EN 2024 в состоянии T6I6 (сердечник соответствует светлой фазе, оболочка - тёмной).

\section{Испытание на усталость сплава PN-EN 2024 в состоянии Т6I6}

Результаты получены в ходе резонансных исследований для выявления предела усталостной прочности 24 образцов сплава PN-EN 2024 в состоянии T6I6. На рис. 8 представлены результаты исследования усталости, на их основе была сделана кривая Wöhler'a с характерными изломами образцов при разных напряжениях. Затем по трем образцам - № 8, 15, 17 - в различных диапазонах напряжений провели анализ изломов и микроструктуры, которые были использованы для определения влияния выделений со структурой сердечник/оболочка на возникновение и развитие усталостного разрушения (рис. 8,9$)$.

Определено, что исходя из зависимости $\mathrm{Z}_{\mathrm{go}}=0,35 \mathrm{R}_{\mathrm{m}}$ оценочное значение усталостной прочности для сплава PN-EN 2024 T6I6 имеет значение около 169,3 МПа, а на кривой Wöhler’a это значение составляет около 200 МПа. Из этого следует, что алюминиевый сплав PN-EN 2024 T6I6 может иметь значение усталостной прочности не менее чем в состоянии T351.

Анализ микроструктуры подтвердил наличие смешанного излома в материале, характер которого зависит от параметров исследования. Усталостное разрушение в образце 17 (область наибольшего напряжения $\sigma=324 \mathrm{MПа)} \mathrm{имеет} \mathrm{пластичный} \mathrm{излом} \mathrm{с} \mathrm{большой} \mathrm{зоной} \mathrm{разрешения,}$ его поверхность имеет характерную форму раковины с видимыми усталостными линиями,

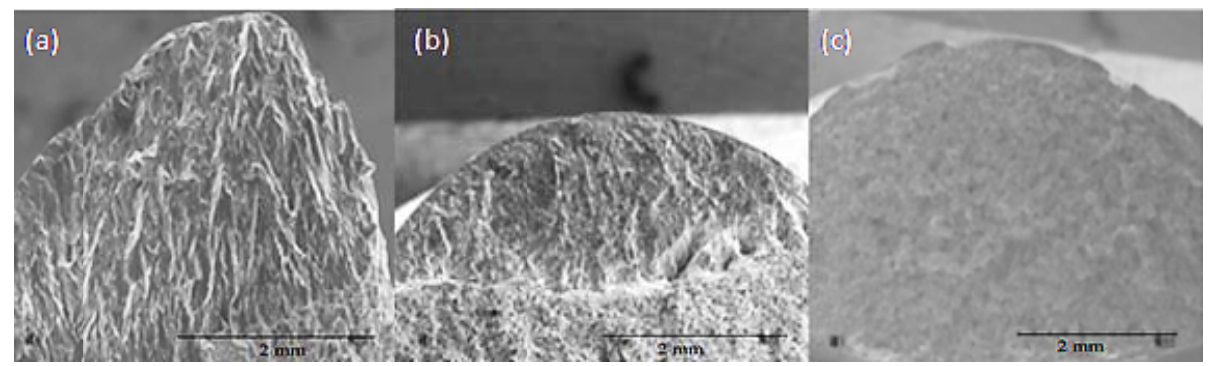

Рис. 9. СЭМ, изломы после испытания на усталостную прочность сплава PN-EN 2024 T6I6: а - образец 17 ; b - образец $15 ;$ с - образец 8

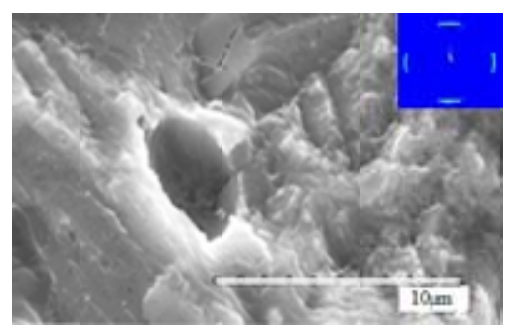

a

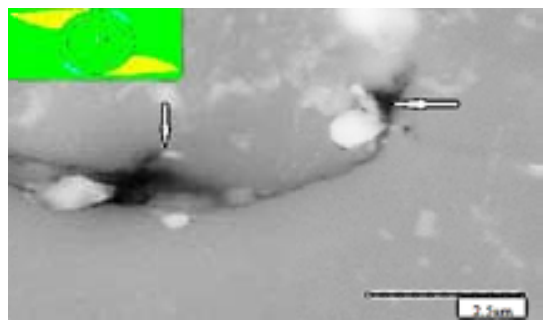

6

Рис. 10. СЭМ, изломы после испытания на усталостную прочность сплава PN-EN 2024 T6I6, образец 15: a - сеть микротрещин вокруг разделения; б - распространение и задержка усталостных трещин 
указывающими на распространение трещин по ступеням (рис. 10a). Тем не менее, в образце 15 (область, соответствующая напряжению неограниченной усталостной прочности $\sigma=207 \mathrm{MПа)}$ излом смешанный, на поверхности разрушение является хрупким, а внутри зона пластичного излома разделена зонами хрупкого разрушения (рис. 10б). Вокруг выделения наблюдается зона пластификации - светлая оболочка (рис. 10a), а в местах превышения предела текучести матрицы вокруг выделения формируется сеть микротрещин. В области косвенных напряжений $\sigma=273$ МПа (образец 8) излом хрупкий (рис. 9c). Проведенные наблюдения показывают, что значительное влияние на усталость имеют только эти выделения со структурой сердечник/ оболочка, которые служат препятствием в местах распространения трещин (рис. 11). Это происходит, когда начало трещины попадает в зону сильных полей напряжений, формируемую вокруг выделения из-за накопления энергии от знакопеременной нагрузки; при этом в результате декогезионного разрушения могут образоваться усталостные пустоты. Благодаря формированию пустот напряжения уменьшаются, а затем происходит процесс релаксации, и распространение трещины может быть остановлено в результате того, что выделение становится местом задержки трещины (стрелка на рис. 11).

Особое внимание при испытании на усталостную прочность уделяется анализу поведения выделений со структурой сердечник/оболочка, лежащих на усталостных линиях фронта, на границе хрупкого и пластичного изломов, а также в области пластичного излома.

На рис. 11 чёрной стрелкой обозначена зона пластификации вокруг выделения, наличие которой приводит к снижению локального напряжения и диссипации энергии распространения микротрещин. В результате усталостные трещины не обнаружены, но показано изменение в направлении усталостных линий фронта в непосредственной близости от выделения. Там же обнаружено нарушение скольжения в плоскости раскола, что может привести к переходу линии скольжения из одной плоскости в другую, с более энергетически выгодным положением.

На рис. 12 представлена поверхность излома с выделениями, лежащими на границе хрупкого и пластичного изломов. Было отмечено, что выделения, прежде чем они были выведены из матрицы, накапливали вокруг себя энергию (следы пластификации отмечены белыми стрелками). Распространение усталостных трещин временно затихло, но после превышения критического напряжения произошло скольжение в непосредственной близости к плоскости спайности и разрушение по ближайшим плоскостям скольжения; одновременно происходит изменение характера излома.
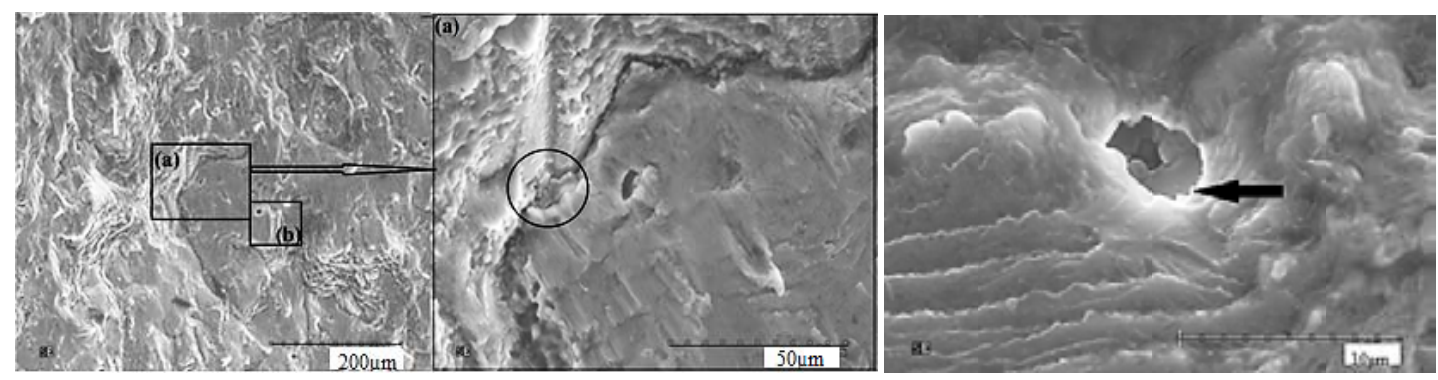

Рис. 11. СЭМ; излом после испытания на усталостную прочность сплава PN-EN 2024 T6I6; образец 15

$$
-76-
$$



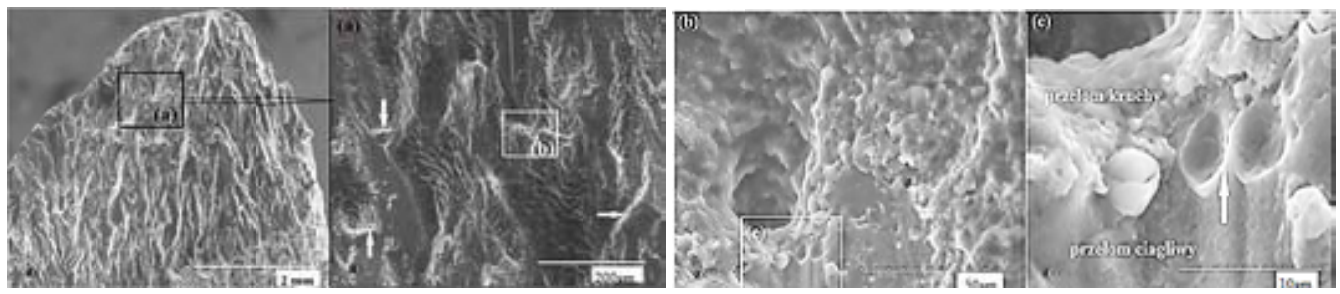

Рис. 12. СЭМ, излом после испытания на усталостную прочность сплава PN-EN 2024 T6I6; образец 17; увеличения (a) отмечены белыми стрелками примеры зон пластификации; $b, c$ - увеличенный вид выделения на границы хрупкого и пластичного характера излома

\section{Испытания на растяжение сплава PN-EN 2024 в состоянии Т6I6}

Для определения значения $\mathrm{R}_{\mathrm{m}}, \mathrm{R}_{02}$ и удлинения A сплава PN-EN 2024 в состоянии T6I6 на 16 образцах проведено испытание на растяжение. На выбранных осевых и радиальных образцах сделан микроанализ изломов, чтобы определить влияние выделений со структурой сердечник/оболочка на упрочнение в процессе осевого растяжения. Для пластичных материалов при значении $\mathrm{R}_{\mathrm{m}}$ наблюдается образование шейки, раскрывается область видимого уменьшения нагрузки и напряжение при разрыве $\mathrm{R}_{\mathrm{B}}$ меньше, чем сопротивление растяжению. На рис. 13 представлена кривая напряжение-деформация, полученная в ходе испытания 16 образцов. Получен график зависимости напряжение-деформация без предела текучести, но с заметной разностью величин $\mathrm{R}_{\mathrm{m}}$ и $\mathrm{R}_{\mathrm{B}}$.

Данная кривая напряжение-деформация подтверждает, что исследуемый материал является пластичным, что доказывает выделенная кругом часть графика - область заметного снижения нагрузки и заметная разница значений $\mathrm{R}_{\mathrm{m}}$ и $\mathrm{R}_{\mathrm{B}}$ (рис. 13). Кроме того, на исследуемых образцах наблюдали образование шейки. Для 8 осевых образцов получено среднее значение $\mathrm{R}_{\mathrm{m}}=513$ МПа и для 8 радиальных $-\mathrm{R}_{\mathrm{m}}=415$ МПа (табл. 2).

При исследовании места разрушения определение наличия выделений на поверхности излома затруднено. Однако на рис. 14 стрелкой (c) показаны места, где они могут быть до разру-

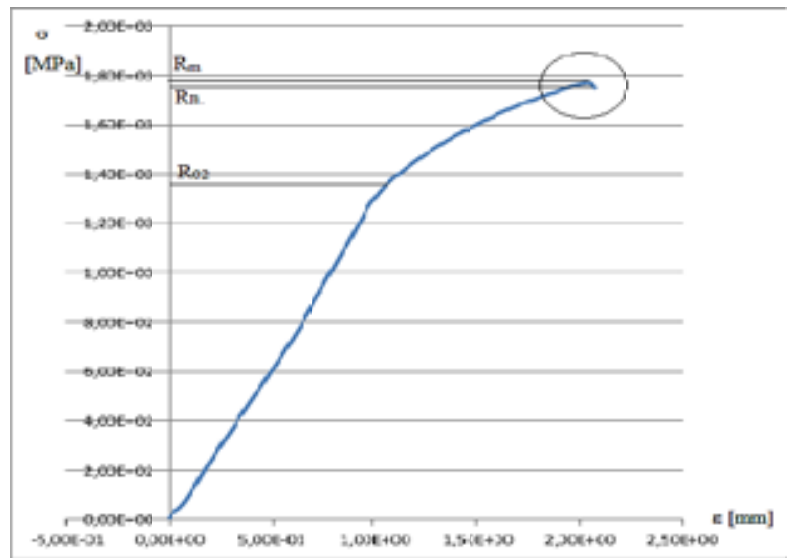

Puc. 13a. Кривая зависимости напряжение - деформация сплава PN-EN 2024 в состоянии Т6I6 


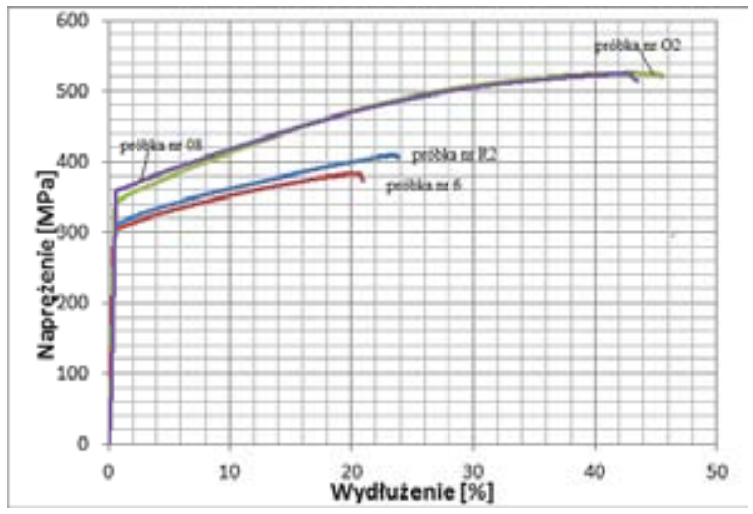

Puc. 13 б. Кривая зависимости напряжение - деформация сплава PN-EN 2024 в состоянии Т6I6; образцы, вырезанные вдоль оси O2 и O8, и образцы, вырезанные в радиальном направлении R2 и R6

Таблица 2. Результаты исследования сопротивления растяжению

\begin{tabular}{|c|c|c|c|c|}
\hline & $\mathrm{R}_{02}[\mathrm{M \Pi а}]$ & $\mathrm{R}_{\mathrm{m}}[\mathrm{M \Pi а}]$ & $\mathrm{R}_{\mathrm{B}}[\mathrm{M \Pi а}]$ & $\mathrm{A}[\%]$ \\
\hline 2024 Т6І6 вдоль оси & 299 & 513 & 504 & 43 \\
\hline 2024 Т6І6 радиально & 222 & 415 & 408 & 22 \\
\hline
\end{tabular}

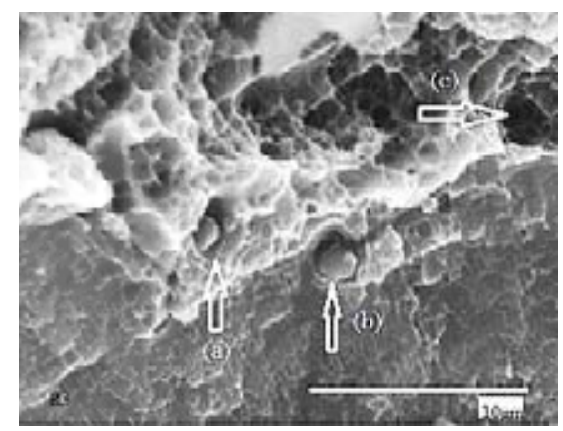

Рис. 14. СЭМ; сплав PN-EN 2024 T6I6; а и b - выделения со структурой сердечник/оболочка; с предположительное место выделения после разрушения

шения. Выделение, указанное стрелкой (a), находится в области хрупкого излома, а указанное стрелкой (b) - пластичного.

Вокруг выделений (независимо от положения) наблюдается неоднородность матрицы, и похоже, что выделения связаны с матрицей локально. Их поверхность (оболочка) выглядела бы непрерывной, если бы трещина не распространялась в матрице или на границе матрица/выделение. СЭМ-изображение выделений, лежащих в области хрупкого разрушения (рис. $15 b$ ), подтверждает предложенную в анализе моделирования (МЭС рис. 15a) возможность образования широкой области зоны пластификации вокруг выделения, в котором после превышения критического напряжения может генерироваться неоднородность материала, в частности вдоль оси 


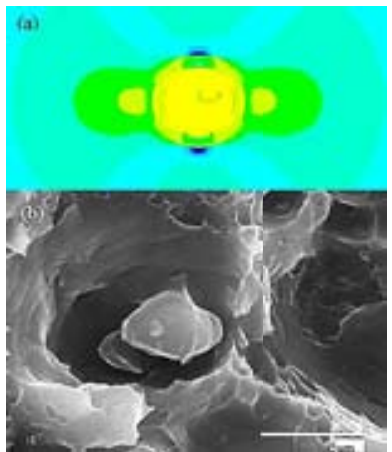

Рис. 15. Выделение в сплаве PN-EN 2024 в состоянии Т6I6: а-симуляция МЭС, распределение напряжений при испытании на растяжение при осевой нагрузке $15 \mathrm{H}$; b - СЭМ- изображение выделения в области хрупкого излома

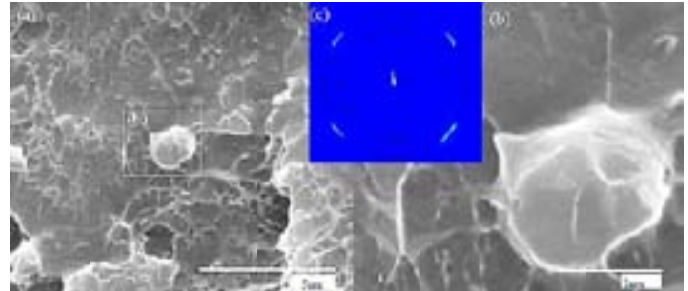

Рис. 16. Выделение в сплаве PN-EN 2024 в состоянии Т6I6: а - зона пластификации в области пластичного излома и вокруг выделения; b выделение увеличение; с - симуляция МЭС

$\mathrm{X}$. В то же время очевидно, что вдоль оси Y на границе матрица/оболочка образовались места с относительно низким напряжением, не подверженные деформации, пластификации и непрерывно связанные с матрицей.

Вокруг выделения, лежащего в области пластичного излома, создается очень тонкая зона пластификации. Следы пластификации также видны по всей зоне пластичного излома (рис. 16a). СЭМ-изображение выделения, лежащего в зоне пластичного излома (рис. 16b), подтверждает предложенную в анализе моделирования (МЭС рис. 16c) возможность только локальной деформации на границе матрица/выделение. Исследование показало, что выделение не подвергается сдвигу во время осевого растяжения, а способствует образованию вокруг него зоны пластификации, которая после превышения критического напряжения в матрице из-за декогезионного разрушения может превратиться в пустоту, и путем дальнейшего повышения нагрузки выделение может быть выведено из матрицы; сопротивление растяжению $\left(\mathrm{R}_{\mathrm{m}}\right)$ при этом повышается. Кроме того, для значения напряжения ниже критического, но выше, чем предел текучести (между $\mathrm{R}_{\mathrm{m}}$ a $\mathrm{R}_{02}$ ), существует пластификация матрицы вокруг выделения, которая может являться источником повышения пластичности для этого материала в состоянии Т6I6.

\section{Выводы}

На основании исследований, проведенных в данной работе, можно сделать следующие выводы:

1. В промышленном сплаве алюминия 2024 в состоянии Т6I6 получены выделения со структурой сердечник/оболочка по второму механизму кинетики образования.

2. Для данного сплава определены следующие свойства: предел выносливости 200 МПа; сопротивление растяжению 513 МПа; предел текучести 299 МПа и удлинение при разрыве $43 \%$.

3. Показано, что механические характеристики алюминиевого сплава 2024 в состоянии T6I6 выше, чем в сплавах со стандартной термообработкой Т3 и Т6.

$$
-79-
$$


4. Оболочка выделения под нагрузкой играет особую роль буфера между мягкой основой и твёрдым сердечником и существенно влияет на упрочнение материала.

Работа выполнена в рамках проекта: „Wplyw wydzieleń typu „rdzeń/powtoka” na wzrost wydtużenia przy podwyższeniu granicy plastyczności stopu aluminium 2024” - NCN 2013/09/b/ ST8/03520.

\section{Список литературы}

1. Fuller C.B., Seidman D.N., Temporal evolution of the nanostructure of Al(Sc,Zr) alloys. Part II-coarsening of Al3(Sc1-xZrx) precipitates. Acta Mater. 53 2005. P. 5415-5428.

2. Kipling K.E., Karnesky R.A., Lee C.P., Durand D.C., Seidman D.N. Precipitation evolution In Al-0,1Sc, Al-0,1Zr and Al-0,1Sc-0,1Zr(at. \%) alloys during isochronal aging. Acta Mater. 58 2010, p. 5184-5195.

3. Ringer S.P., Muddle B.C., Polmear I.J., Effects of Cold Work on Precipitation in Al-Cu-Mg and Al-Cu-Li-(Mg-Ag) Alloys. Metallurgical and Materials Trans. A v. 26 A VII 1995, p. 1659.

4. Chinh N.Q., Gubicza J., Czeppe T., Lendvai J., Xu C., Valiev R., Langdon R.Z. Developing a strategy for the processing of age-hardenable alloys by ECAP at room temperature. Mat. Sc. and Eng. A 516 2009, p. 248-252.

5. Mahallawy N.E., Shehata F.A., Hameed M.A.E., Kim H.S., 3D FEM simulations for the homogeneity of plastic deformation in Al-Cu alloys during ECAP. Mat. Sc. and Eng. A 5272010. P. 1404-1410.

6. Kaczmarek Ł., Stegliński M., Radziszewska H., Sawicki J., Kozioł K., Gawroński Z. Иcпользование двухстадийного старения для повышения прочностных свойств сплава PN-EN 2024: ХІІ Международная научно-техническая уральская школа-семинар металловедов-молодых ученых. Екатеринбург 2011. [Kaczmarek Ł., Stegliński M., Radziszewska H., Sawicki J., Kozioł K., Gawroński Z. Using the two step aging for strength improving of PN-EN 2024 alloy. XII International science and technical Ural school-seminar of metal scientists. Yekaterinburg 2011 (in Russ.)]

7. Hansen V., Vevecka-Priftaj A., Fjerdingen J., Langsrud Y., Gji J., The Influence of Silicon on Age Hardening Kinetics and Phase. Mat. Sc. Forum V. 519-521 2006. P. 576-584.

8. Shan Z.H., Wang Z.G. Zhang Y. Effect of notch geometry on short fatigue crack growth in 8090 Al-Li alloys. J. of Mat. Sc. 32 1997. P. 4673-4677.

9. Tkaczyk S., Kciuk M., Zmiana własności mechanicznych i korozji naprężeniowej w różnych środowiskach stopu aluminium typu AlMg5. 12 International Scientific Conference Achievements in mechanical and materiale engineering. Gliwice, 2003.

10. Senderski J., Płonka B., Kłyszewski A., Stuczyński T. Kucie matrycowe stopów aluminium serii 2XXX, 4XXX przy zastosowaniu materiału wsadowego w różnej postaci. Ob. Pl. Met. t. XVIII nr 12007.

11. Белов Н.А. Экономнолегированные высокопрочные алюминиевые сплавы - принципы оптимизации фазового состава. МиТОМ 2011, № 9, 19-27. [Belov N.A. Sparingly doped highstrength aluminium alloys - principles of phase composition optimization. MiTOM 2011 (9). P. 19-27 (in Russ.)] 
12. Дриц А.М., Овчинников В.В. Свариваемость и свойства сварных соединений высокопрочных алюминиевых сплавов системы Al.-Cu-Li. MuTOM 2011, № 9, 45-50. [Dritz A.M., Ovchinnikov V.V. Weldability and properties of welded joints of high-strength aluminum alloys of system Al-Cu-Li. MiTOM 2011 (9), 45-50 (in Russ.)]

13. Лопатин Ю.Г., Чувильбеев В.Н., Нохрин А.В., Копылов В.И., Пирожникова О.Э., Влияние температуры интенсивной пластической деформации на предел диспергирования зерен в металлах и сплавах. Вестник Нижегородского университета 2010, №5 (2), 132-137. [Lopatin Yu.G., Chuvilbeev V.N., Nokhrin A.V., Kopylov V.I., Pirozhnikova O.E. Effect of intensive plastic deformation temperature on grain dispersement limit in metals and alloys. Journ. of Nizhn. univ. 2010, 5(2), 132-137 (in Russ.)]

14. Wierszyłłowski I., Wieczorek S., Stankowiak A., Samolczyk J. Kinetyka przemian podczas przesycania i starzenia stopu Al.-4,7 \%Cu: Ob. Pl. Met. nr5 2005.

15. Waheed A. Dispersoids in Al-Li AA 8090 series alloys. J. of Mat. Sc. 32 1997. P. 3341-3347.

16. Wierszyłłowski I. Relacje pomiędzy procesami starzenia i odkształcenia stopu Al-4,7 \%Cu. Badania twardości i DTA. Ob. Pl. Met t. XVII nr 12006.

17. Tkaczyk S., Kciuk M., Odporność korozyjna stopu AlMg1Si1. 11 International Scientific Conference Achievements in mechanical and materiale engineering. Gliwice, 2002.

18. Батышев К.А. Литье с кристаллизацией под давлением алюминиевых сплавов. Часть 1. МиТОМ 2011. № 10, 3-11. [Batyshev K.A. Casting of aluminium alloys with solidification under pressure. Part 1, MiTOM 2011. (10), 3-11 (in Russ.)]

19. ] Radmilovic V., Tolley A., Marquia E.A., Rossell M.D., Lee Z., Dahmen U. Monodisperse Al3(LiScZr) core/shell precitates in Al alloys. Sc. Mat. 58 2008, P. 529-532.

20. Lertora E., Gambaro C., AA 8090 Al-Li alloy FSW Parameters to minimize defects and increase fatigue life. Int. Mater. Form v. 3 Suppl. 1 2010. P. 1003-1006.

21. Lee E.W., Kalu P.N., Brandao L., Es-Said O.S., Foyos J., Garmestani H. The effect of off-axis thermomechnical processing on the mechanical behavior of textured 2095 Al-Li alloys. Mat. Sc. and Eng. A 265. 1999. P. 100-109.

22. Tóth L., Lapovok R., Hasani A. Gu C. Non-equal channel angular pressing of aluminum alloy. Sc. Mat. 61. 2009. P. 1121-1124. 